1 Lou Ruvo Center for Brain Health, Cleveland Clinic Nevada, Nevada, USA

2 Centre of Excellence in Neurodegeneration, Centre

Hospitalier Universitaire de Toulouse, Toulouse, France

Correspondence to: J Shi SHIJ3@ccf.org Cite this as: BMJ 2020;371:m3684 http://dx.doi.org/10.1136/bmi.m3684 Published: 09 October 2020
BRAIN HEALTH

\section{Alzheimer's disease beyond amyloid: strategies for future therapeutic interventions}

\author{
Jiong Shi and colleagues discuss recent evidence of clinical trials for Alzheimer's disease and new \\ development strategies
}

Jiong Shi, ${ }^{1}$ Marwan N Sabbagh, ${ }^{1}$ Bruno Vellas ${ }^{2}$

Neurodegenerative diseases encompass a variety of medical conditions that affect the survival and function of neurons in the brain. Neuronal loss often results in a decline of cognitive function and advances to dementia. Dementia is the common denominator of neurodegenerative diseases. The World Health Organization estimated that the number of people living with dementia worldwide in 2015 was 47.47 million. As the population ages, this number is expected to reach 75.63 million in 2030 and 135.46 million in 2050. Alzheimer's disease is the most common cause of dementia in older people. The natural course of dementia, particularly Alzheimer's disease, results in significant disability and dependence. The effect on care givers and the public health system is staggering. The total estimated costs of dementia were $\$ 604 \mathrm{bn}$ ( $€ 471 \mathrm{bn}$; $€ 519 \mathrm{bn}$ ) in 2010, roughly $1 \%$ of the world's gross domestic product (www.who.int). No disease modifying treatment exists for dementia.

\section{Pathology}

Alzheimer's disease is characterised by progressive memory decline and deficits in at least one other cognitive domain that significantly impairs normal occupational and social function. ${ }^{1}$ Pathologically, in addition to neuronal and synaptic loss, the disease is defined by pathological hallmarks-namely, amyloid $\beta$ accumulation as diffuse and neuritic plaques and hyperphosphorylated tau protein in the form of neurofibrillary tangles. ${ }^{2}$

Amyloid $\beta$ peptide was first sequenced in 1984 and later identified as the main component of neuritic plaques. ${ }^{34}$ Amyloid $\beta$ is a product of the larger transmembrane amyloid precursor protein. Acting together, $\beta$-secretase and $\gamma$-secretase break down and slice amyloid precursor protein into smaller fragments. Amyloid $\beta$ monomers are formed by backbone hydrogen bonds between their $\beta$ strands. ${ }^{5}$ These monomers are prone to misfolding and are considered critical to the neurodegenerative process. The misfolded proteins trigger production of further misfolded proteins, which accumulate into aggregates or plaques.

Human genetic studies on autosomal dominant early onset familial Alzheimer's disease have shown that mutations in one of the three genes encoding amyloid precursor protein, presenilin 1 , or presenilin 2 result in increased production of amyloid $\beta$. Apolipoprotein $\mathrm{E} \varepsilon 4$ allele is the strongest genetic risk factor for late onset sporadic Alzheimer's disease. It increases the risk of the disease by three to four times in heterozygotes and by around 12 times in homozygotes. The apolipoprotein E $\varepsilon 4$ allele has been shown to reduce the clearance, and increase the seeding, of amyloid $\beta .^{6}$ These data from human genetic studies led to the hypothesis of the amyloid cascade. ${ }^{7}$ Evidence from transgenic amyloid $\beta$ mouse models provided mechanistic support for the hypothesis, in which amyloid $\beta$ accumulation is the critical initial step in the pathogenesis of Alzheimer's disease. ${ }^{8}$ Amyloid $\beta$ triggers subsequent hyperphosphorylation and accumulation of tau protein, neuronal and synaptic loss, and, ultimately, results in clinical symptoms. ${ }^{7}$

Additionally, Alzheimer's disease is a tauopathy as shown by abnormal levels of hyperphosphorylated tau protein. The pathological aggregation of these proteins lead to neurofibrillary tangles. The non-pathological tau protein is involved in stabilising microtubules, which make up the cytoskeleton of the cell. When the tau protein is hyperphosphorylated, it induces the breakdown of microtubules and the formation of insoluble aggregates of neurofibrillary tangles in the brain. In Alzheimer's disease, neurofibrillary tangles emerge from the internal brain structures to more distal regions-namely, from the transentorhinal cortex to the hippocampus and then after tau pathology manifests in the neocortex. ${ }^{10}$ In contrast to amyloid $\beta$, tau pathology has shown a strong correlation with declining cognitive performance based on longitudinal pathological and imaging studies. ${ }^{11}$

\section{Pathological hallmark targeted clinical trials for Alzheimer's disease}

During the past two decades, treatments targeting amyloid $\beta$ have been designed to lower amyloid $\beta$ concentrations and prevent the amyloid $\beta$ triggered cascade. Several compounds have been developed to target various forms of amyloid $\beta$, monomeric, oligomeric, aggregates, and plaques. The initial trial (AN1792) attempted to achieve active immunisation by injections of a full length amyloid $\beta$ peptide in patients with Alzheimer's disease. This study was terminated early owing to complications of encephalomeningitis. ${ }^{12}$ Additional treatments were designed to increase amyloid $\beta$ clearance from the brain, including inoculations with amyloid $\beta$ antigens (ABvac40, CAD-106), anti-amyloid $\beta$ monoclonal antibodies (bapineuzumab, solanezumab, and the neocortex. ${ }^{9}$ Cognitive impairment is evident only 
crenezumab), and anti-amyloid $\beta$ polyclonal antibodies (immunoglobulins). Trials to decrease the production and aggregation of amyloid $\beta$ included amyloid $\beta$ aggregation inhibitors (tramiprosate, scyllo-inositol, PBT2), $\gamma$-secretase inhibitors (avagacestat and semagacestat), $\gamma$-secretase modulators (tarenflurbil), and $\beta$ site amyloid precursor protein cleaving enzyme inhibitors (LY2886721, umibecestat, elenbecestat, verubecestat, atabecestat, and lanabecestat). No clinical efficacy was shown in any of these studies. The $\beta$ site amyloid precursor protein cleaving enzyme inhibitors worsened cognitive function, probably because they have other functions critical to neuronal development.

Furthermore, the use of $\gamma$-secretase inhibitors caused adverse effects because of their function on Notch signalling pathways.

Recognition of the clinical significance of tau pathology in comparison with amyloid $\beta$ has resulted in a resurgence of interest in targeting tau protein. Treatments designed to inhibit production of phosphorylated tau protein have been investigated.

Leuco-methylthioninium bis(hydromethanesulphonate) is a methylene blue derivative that reduces fibrillation and aggregation of tau protein. Tideglusib is a glycogen synthase kinase 3 inhibitor that blocks tau kinase and thus the abnormal hyperphosphorylation of tau protein. Neither of these treatments was found to be clinically efficacious. Immunotherapies targeting tau protein (AADvac-1,

ACI-35, BIIB092, and ABBV-8E12) are currently in phase II trials for patients with early Alzheimer's disease..$^{13}$ ABBV-8E12 has already been studied in patients with progressive supranuclear palsy. Progressive supranuclear palsy is considered a "pure" tauopathy because it has abundant tau pathology but lacks amyloid pathology. This study of progressive supranuclear palsy was terminated early owing to ineffectiveness.

\section{Analysis of clinical trials targeting biomarkers}

Several reasons have been proposed for the lack of efficacy of pathology targeted treatment in clinical trials. Firstly, it has been suggested that the dose used to affect the disease could be inadequate or result in unacceptable adverse effects. One of the earliest clinical trials (AN1792) was effective in reducing amyloid $\beta$ concentrations in the brain. Unacceptable levels of encephalomeningitis developed, however, and the trial was terminated. This set the tone for future investigations, and subsequent trials focusing on amyloid $\beta$ immune treatments have used doses to minimise the adverse effects rather than maximise the benefits. Many trial regimens were terminated early as a precaution when there was evidence of inflammatory changes or microhaemorrhages in the brain, although some argued that this could have been early evidence of the immunotherapy efficacy rather than a risk of future encephalitis. Additionally, the human blood-brain barrier is much more discriminating than that of lower species. Therefore, higher concentrations may be required to achieve the therapeutic effect seen in animal models. For instance, solanezumab penetration into the central nervous system is only $0.1 \%$ to $0.3 \%$ of the concentration measured in plasma. Aducanumab and gantenerumab showed a better and more clinically meaningful outcome when higher doses were used. ${ }^{14}$

Secondly, considerable controversy exists about the stage at which Alzheimer's disease is reversible. Most of the failed phase III trials enrolled patients with mild to moderate disease. Longitudinal clinical imaging/pathological correlation studies have disclosed a preclinical phase of Alzheimer's disease that precedes the onset of symptoms by a couple of decades. ${ }^{15}$ It has been hypothesised that the onset of pathological deposition is the time when the disease may be amenable to immune treatments. Thus several large scale prevention trials, such as the Anti-Amyloid Treatment in
Asymptomatic Alzheimer's (A4) trial, Alzheimer Prevention Initiative (API), and the Dominantly Inherited Alzheimer Network Trial (DIAN-TU), were designed to capture patients in the presymptomatic stage. These clinical trials test anti-amyloid treatments in cognitively normal patients who are at high risk for developing Alzheimer's disease.

Thirdly, amyloid $\beta$ and tau protein may synergistically and simultaneously cause pathological changes in Alzheimer's disease. Amyloid $\beta$ enhances phosphorylation, truncation, and aggregation of tau protein, whereas tau protein further induces the production of amyloid $\beta$ species. The effective suppression of only one of these two factors is probably insufficient to produce a clinical benefit. Thus an approach targeting them simultaneously or sequentially may be necessary to affect the course of the disease.

Finally, although the amyloid hypothesis and tau pathology are supported by considerable genetic and biomarker studies, the data from failed clinical trials suggest that other potential targets should be explored.

\section{Non-biomarker targets}

In most patients, Alzheimer's disease is late onset, and the most common risk factor for Alzheimer's disease is ageing. Ageing in the industrialised world is associated with cardiovascular and cerebrovascular diseases, as well as an increased incidence of risk factors such as hyperlipidaemia, hypertension,

hyperhomocysteinaemia, and diabetes mellitus. Autopsy studies have shown that more than half of patients with Alzheimer's disease have mixed vascular pathology. Amyloid $\beta$ generates reactive oxygen species, which cause capillary constriction in the human cortex, resulting in reduced cerebral blood flow. Hypoxia can also increase amyloid $\beta$ production, thus generating a vicious cycle. Vascular impairment can ultimately lead to hypoperfusion, oxidative stress, inflammation, and dysfunction of the neurovascular unit. The Systolic Blood Pressure Intervention Trial-Memory and Cognition in Decreased Hypertension (SPRINT-MIND) showed that intensive systolic blood pressure control reduced the incidence of mild cognitive impairment. The Finnish Geriatric Intervention Study to Prevent Cognitive Impairment and Disability (FINGER) focused on the treatment of modifiable vascular and lifestyle related risk factors. The intervention was effective in reducing the risk of cognitive decline. Similarly, the Multidomain Alzheimer Preventive Trial (MAPT) has shown that a multidomain intervention alone or in combination with omega-3 fatty acids improved cognitive outcome in subjects with positive amyloid status. ${ }^{16}$ The validity of these results will be further tested by the worldwide FINGERS network, which shares core methodology with consideration of local culture and adaptations. Hyperhomocysteinaemia is a modifiable risk factor for cognitive impairment. The relative risk of dementia is up to 2.5 in older people with moderately raised homocysteine. Homocysteine lowering treatment with B vitamins could slow down the rate of brain atrophy and cognitive decline. ${ }^{17}$

The role of inflammation, also significant in cerebrovascular disease and ageing, has been studied for its effect on Alzheimer's disease. Systemic inflammatory markers such as $\mathrm{C}$ reactive protein and interleukin 6 are associated with neuronal and synaptic loss and poor cognitive performance in older people. ${ }^{18}$ However, anti-inflammatory treatment, including low dose prednisone, low dose aspirin, non-steroidal anti-inflammatory drugs, selective cyclo-oxygenase-2 inhibitors, and etanercept, has failed to show clinical efficacy in patients with mild to moderate and preclinical Alzheimer's disease. One explanation for the lack of efficacy of systemic anti-inflammatory agents could be their poor penetration 
across the blood-brain barrier. In the central nervous system, there is a growing body of evidence linking the role of immunogenicity to pathology and the clinical manifestations of Alzheimer's disease.

Whole genome analysis studies have disclosed the evidence leading to clinical trials. They identified several immune related genetic risk factors which may contribute to the inflammatory process and increased cytokine production in Alzheimer's disease, ${ }^{19}$ including triggering receptor expressed on myeloid cells 2 (TREM2) and CD33. Both are involved in microglial activation, cytokine production, and inflammation. The TREM2 activating antibody and CD33 blocking antibody are in phase I trials. Although microglial activation is related to the pathology of Alzheimer's disease, further work is needed to examine the Janus faced effects of microglia and associated cytokines. Furthermore, increasing evidence has shown that interactions between the gut microbiome and the central nervous system innate immune system (gut-brain axis) may be involved in the pathogenesis of Alzheimer's disease. Microglial activation and function are regulated by the microbiome via microbiome derived metabolites. ${ }^{20}$

\section{Conclusion}

Biomarkers for Alzheimer's disease have been clearly identified yet the disease remains a complex and multifaceted disorder. Few would argue against the value of biomarkers for diagnosis and tracking the course of Alzheimer's disease. As our understanding of these biomarkers advances, however, the limitation of these signature proteins as targets of treatment also emerges. We have delineated several promising strategies, which may improve the clinical outcome of future trials. Firstly, the use of higher concentrations of monoclonal treatments to adjust for poor penetration of the blood-brain barrier; secondly, early identification and treatment of patients at high risk for Alzheimer's disease, and targeting treatment at asymptomatic patients with limited biomarker deposition; and finally, the simultaneous or sequential targeting of both biomarkers with monoclonal treatment to determine whether synergy is needed to achieve efficacy. In the meantime, we need to explore promising areas of research that target pathological changes associated with Alzheimer's disease and affect cognitive performance, such as vascular and inflammatory risk factors. Whether or not these cognitive benefits directly correlate with, or significantly affect, biomarker deposition requires further investigation. Cerebrovascular parameters, which may affect clinical outcome in monoclonal trials, should be evaluated. The combination of cerebrovascular risk modification and monoclonal treatments targeting amyloid $\beta /$ tau protein could have a therapeutic effect not seen in isolation.

\section{Key messages}

- Alzheimer's disease has distinct pathological hallmarks, but clinical trials targeting these biomarkers have failed, indicating the need for new treatment strategies

- Treatment modifications targeting biomarkers include the use of higher concentrations of therapeutic compounds, early identification and treatment of patients at high risk for Alzheimer's disease, and simultaneous or sequential targeting of both amyloid $\beta$ and tau protein

- Promising non-biomarker targeted strategies include modification of cerebrovascular risk factors and targeting inflammatory factors associated with Alzheimer's disease

- Combination therapy of cerebrovascular risk modification and use of monoclonal therapies targeting amyloid $\beta /$ tau protein could have a therapeutic effect.
Contributors and sources: JS, MNS, and BV are experts in clinical research on Alzheimer's disease and related cognitive disorders. This article arose from discussion with The BMJ editors on Brain Health series. JS is the guarantor.

Competing interests: We have read and understood BMJ policy on declaration of interests and have the following interests to declare: JS is the scientific board member of Roche. MNS is the scientific board member of Biogen, Signant Bracket, Eisai, Alzheon, Athira, NeuroReserve, Stage 2 Innovation, VTV Therapeutics, Regeneron, Neurotrope, and Roche-Genentech. BV is the scientific board membe of Acadia, Axome Therapeutics, Biogen, Cerecin, Green Valley Pharmaceuticals, Lilly, MSD, Nestlé, Roche, and Sunovion Pharmaceuticals.

Provenance and peer review: Commissioned; externally peer reviewed.

This article is part of a series launched at the Chinese Stroke Association annual conference on 10 October 2020, Beijing, China. Open access fees were funded by the National Science and Technology Major Project. The BM/ peer reviewed, edited, and made the decision to publish these articles.

This study is supported by NIH COBRE, 5P20GM109025, R01AG059008, and the Keep Memory Alive Foundation.

McKhann GM, Knopman DS, Chertkow H, etal. The diagnosis of dementia due to Alzheimer's disease: recommendations from the National Institute on Aging-Alzheimer's Association workgroups on diagnostic guidelines for Alzheimer's disease. Alzheimers Dement 2011;7:263-9. doi: 10.1016/j.jalz.2011.03.005

2 Jack CR, JrBennett DA, Blennow K, etal. NIA-AA research framework: toward a biological definition of Alzheimer's disease. Alzheimers Dement 2018;14:535-62. doi: 10.1016/j.jalz.2018.02.018 Glenner GG, Wong CW. Alzheimer's disease: initial report of the purification and characterization of a novel cerebrovascular amyloid protein. Biochem Biophys Res Commun 1984;120:885-90. doi: 10.1016/S0006-291X(84)80190-4

4 Masters CL, Simms G, Weinman NA, etal. Amyloid plaque core protein in Alzheimer disease and Down syndrome. Proc Natl Acad Sci USA 1985;82:4245-9. doi: 10.1073/pnas.82.12.4245

5 Soto C, Estrada L, Castilla J. Amyloids, prions and the inherent infectious nature of misfolded protein aggregates. Trends Biochem Sci 2006;31:150-5. doi: 10.1016/j.tibs.2006.01.002

6 Polvikoski T, Sulkava R, Haltia M, etal. Apolipoprotein E, dementia, and cortical deposition of beta-amyloid protein. N Engl J Med 1995;333:1242-7. doi: 10.1056/NEJM199511093331902

7 Hardy J, Allsop D. Amyloid deposition as the central event in the aetiology of Alzheimer's disease. Trends Pharmacol Sci 1991;12:383-8.doi: 10.1016/0165-6147(91)90609-V

8 Games D, Adams D, Alessandrini R, etal. Alzheimer-type neuropathology in transgenic mice overexpressing V717F beta-amyloid precursor protein. Nature 1995;373:523-7. doi: $10.1038 / 373523 \mathrm{a} 0$

9 Braak H, Braak E. Neuropathological stageing of Alzheimer-related changes. Acta Neuropathol 1991;82:239-59.doi: 10.1007/BF00308809

10 Price JL, Morris JC. Tangles and plaques in nondemented aging and "preclinical" Alzheimer's disease. Ann Neurol 1999;45:358-68.

doi: 10.1002/1531-8249(199903)45:3<358::AID-ANA12>3.0.CO;2-X

11 Aschenbrenner AJ, Gordon BA, Benzinger TLS, etal. Influence of tau PET, amyloid PET, and hippocampal volume on cognition in Alzheimer disease. Neurology 2018;91:e859-66. doi: 10.1212/WNL.0000000000006075

12 Holmes C, Boche D, Wilkinson D, etal. Long-term effects of Abeta42 immunisation in Alzheimer's disease: follow-up of a randomised, placebo-controlled phase I trial. Lancet 2008;372:216-23. doi: 10.1016/S0140-6736(08)61075-2

13 Gauthier S, Aisen PS, Cummings J, etal. Non-amyloid approaches to disease modification for Alzheimer's disease: an EU/US CTAD Task Force Report. J Prev Alzheimers Dis 2020:1-6. doi: 10.14283/jpad.2020.18

14 Aisen PS, Cummings J, Doody R, etal. The future of anti-amyloid trials. J Prev Alzheimers Dis 2020;7:146-51. doi: 10.14283/jpad.2020.24

15 Vermunt L, Sikkes SAM, van den Hout A, etal. Duration of preclinical, prodromal, and dementia stages of Alzheimer's disease in relation to age, sex, and APOE genotype. Alzheimers Dement 2019;15:888-98. doi: 10.1016/j.jalz.2019.04.001

16 Delrieu J, Payoux P, Carrie I, etal. Multidomain intervention and/or omega-3 in nondemented elderly subjects according to amyloid status. Alzheimers Dement 2019;15:1392-401. doi: 10.1016/j.jalz.2019.07.008

17 Smith AD, Refsum H, Bottiglieri T, etal. Homocysteine and dementia: an international consensus statement. J Alzheimers Dis 2018;62:561-70. doi: 10.3233/JAD-171042

18 Marsland AL, Gianaros PJ, Kuan DC, etal. Brain morphology links systemic inflammation to cognitive function in midlife adults. Brain Behav Immun 2015;48:195-204. doi: 10.1016/j.bbi.2015.03.015

19 Greig NH, Mattson MP, Perry T, etal. New therapeutic strategies and drug candidates for neurodegenerative diseases: p53 and TNF-alpha inhibitors, and GLP-1 receptor agonists. Ann N Y Acad Sci2004;1035:290-315. doi: 10.1196/annals.1332.018

20 Wang $X$, Sun G, Feng T, etal. Sodium oligomannate therapeutically remodels gut microbiota and suppresses gut bacterial amino acids-shaped neuroinflammation to inhibit Alzheimer's disease progression. Cell Res 2019;29:787-803. doi: 10.1038/s41422-019-0216-X 


\section{ANALYSIS}

This is an Open Access article distributed in accordance with the Creative Commons Attribution Non Commercial (CC BY-NC 4.0) license, which permits others to distribute, remix, adapt, build upon this work non-commercially, and license their derivative works on different terms, provided the original

work is properly cited and the use is non-commercial. See: http://creativecommons.org/licenses/by$n c / 4.0 \%$ 